\title{
La théorie de l'électricité de Nollet et son application en médecine à travers sa correspondance inédite avec Jallabert
}

Par Isaac Benguigui

\section{Les deux savants}

D'origine modeste, Jean-Antoine Nollet naquit le 19 novembre 1700 à Pimprez $^{1}$. Après des études de théologie, il devenait diacre en 1728. Mais la théologie ne semble pas être sa voie. Membre de la Société des Arts, où il rencontre plusieurs savants, deux illustres physiciens lui demandent de se joindre à leurs travaux. D'abord Du Fay, qui fera appel aux dons de Nollet pour ses expériences en électricité, à laquelle il doit attacher son nom, ensuite Réaumur, dont il sera pendant plusieurs années le fidèle collaborateur, l'élève et l'ami. Son voyage à Londres est couronné par son élection à la Royal Society.

En 1735, Nollet est chargé d'organiser un cours de physique expérimentale et de le professer. Son but est d'introduire dans les esprits le goût de la science. Son enseignement est si apprécié qu'il sera invité à donner des cours dans plusieurs académies. En 1741, c'est l'Académie de Bordeaux qui fera appel à son talent, et la République de Berne le chargera de créer une école de physique expérimentale et de former le futur professeur M. Niklaus Blauner qui suit ses cours (1750). Professeur au Collège de Navarre, où il aura «jusqu'à 800 auditeurs», il sera nommé par le roi maître de physique et d'histoire naturelle des Enfants de France, et professeur à l'Ecole militaire de Mézières ${ }^{2}$. Son activité à l'Académie n'a jamais cessé d'être considérable. Ses travaux sont d'une grande diversité. En 1770, il fut nommé adjointdirecteur de l'Académie.

Jean Jallabert (1712-1768) ${ }^{3}$ a été l'élève de Gabriel Cramer et Jean-Louis Calandrini. Jean-Alphonse Turrettini l'engage à faire des études de théologie, ce qu'il accepte, et, en 1737, il sera reçu au saint-ministère. La mort de Turrettini et l'insistance de Cramer et de Calandrini font que la vocation de Jallabert se tourne vers les sciences. Les magistrats de Genève créent en sa faveur la première chaire de physique expérimentale. 
Mais avant d'exercer ses fonctions, il effectuera un séjour en Suisse et à l'étranger au cours duquel il va nouer des contacts avec plusieurs savants et acquérir les instruments nécessaires pour monter un cabinet de physique. Il se rendra tout d'abord à Bâle, où il fréquentera les Bernoulli, en particulier Daniel, l'auteur de l'Hydrodynamica, dont la publication est imminente (1738), puis en Hollande et en Angleterre. Enfin à Paris où il rencontre Réaumur, Buffon et l'Abbé Nollet. Dès cet instant, une amitié et une collaboration s'installent entre lui et ce dernier.

En 1739, il donne sa leçon inaugurale. Son discours traite de l'utilité de la philosophie expérimentale (entendre la physique). Daniel Bernoulli qui en a reçu un imprimé lui écrit: «Je prévois que vous allez faire grand bruit dans l'Europe savante; vous êtes fait pour éclairer le monde.» ${ }^{4}$

Nommé quelque temps après conservateur de la Bibliothèque publique de Genève, il s'occupera particulièrement du Département des Manuscrits. Mais le domaine qui passionnera Jallabert est l'électricité. Il jouera également un rôle dans la vie publique: il sera en effet Syndic de la République, de 1765 jusqu'à sa mort en 1768.

\section{La correspondance}

Cette correspondance s'ouvre sur une lettre de Nollet datée du 2 octobre 1739 et se termine sur une lettre datée du 30 juillet 1760 . Elle comporte 45 lettres inédites de Nollet et quelques minutes de Jallabert. Quelques lettres se sont certainement égarees, couvrant la période 1760-1768. Le ton et le style de la dernière lettre n'annoncent aucune rupture.

On peut distinguer dans la correspondance échangée entre les deux grands savants, essentiellement deux périodes:

La première (1739-1744), qui s'étend de la prise de contact entre les deux hommes jusqu'à la lecture du premier mémoire de Nollet à l'Académie ${ }^{5}$. Elle traite de sujets divers et d'ordre général: envoi d'instruments, pompe pneumatique, fabrication de thermomètres, figure de la terre. Nollet y parle de ses ouvrages, qui ont été largement diffusés en Europe, de ses cours et de ses brillantes démonstrations; il s'explique sur le sens de ses livres et le public auquel ils sont destinés. Dans sa lettre de janvier 1744, en expédiant deux exemplaires de ses Leçons de physique 6 (tom. I et II), Nollet demande à Jallabert une certaine indulgence: 
«Lisez la préface affin dentrer dans lesprit qui a conduit ma plume ${ }^{7}$; si javois écrit pour une école publique, et que cette école eut été placée à Leyde, à Genève ${ }^{8}$, etc... j'aurois pris un autre ton, mais à Paris jay plus souvent affaire aux gens du monde.»

\section{Sur sa philosophique scientifique, il déclare:}

«Je suis exactement newtonnien, c'est-à-dire que je ne reconnois lattraction que comme un fait, et que la vertu attractive n'est pas de mon goût.»

La seconde période s'étend de 1745 à 1760. Nollet s'engage dans ses recherches sur l'électricité où ses principes sont exposés d'une manière détaillée. Dans sa lettre du 19 juillet 1745, il déclare:

«Voicy les principes d'expériences sur lesquels je m'appuye:

1) L'électricité est l'action d'une matière qui est en mouvement autour des corps qui deviennent électriques. Car ce qui touche jusqu'à causer de la douleur, ce qui se fait entendre, ce qui a de l'odeur est vraiment une substance matérielle. Vous connaissez tout ce qui peut prouver la mineur[e] de ce syllogisme.

2) Cette matière n'est point l'air grossier que nous respirons; car elle passe à travers du verre et des autres corps solides, que l'air de l'atmosphère ne peut pénétrer.

3) Cette matière est celle du feu et de la lumière; car elle présente en tout temps et en tout lieu au dehors et au dedans des corps, comme le feu élémentaire; elle n'a besoin que d'être excitée pour paroître.

4) Mais le feu élémentaire faisant fonction de matière électrique n'est pas seul. Il est uni aux parties les plus subtiles des corps mixtes d'où il sort et dans lesquels il reçoit son mouvement.

5) La matière électrique vient non seulement du corps électrisé; mais aussi de tout ce qui l'environne jusqu'à une certaine distance, soit milieu fluide, soit corps solides.

6) La matière électrique sort toujours du corps électrisé en forme d'aigrettes. Elle se meut avec plus de facilité dans les corps solides que dans l'air même.

7) La matière électrique étant au fond la même que celle de la lumière et du feu, doit être considérée comme un fluide qui est présent partout, et qui tendant à se mettre en équilibre avec luy même, s'empresse de remplir les espaces qui se trouvent vides des parties de son espèce... ${ }^{9}$

Après avoir établi ces principes, Nollet range les phénomènes de l'électricité en deux classes:

«L'une contiendra les effets où il y a inflammation et lumière; dans l'autre classe, nous comprendrons les mouvements alternatifs qu'on a nommés attractions et répulsions.»

\section{Pour lui:}

«La matière électrique déterminée par le frottement à sortir de l'épaisseur du verre, y laisse un vuide qui se remplit aussi tost par un fluide de la même espèce, dont il est environné de toutes parts.» 
«Voila donc la matière électrique, qui se meut en deux sens opposés et qui forme pour ainsi dire deux courants. L'un qui sort du tube par des lignes divergentes qu'on appellera matière effluente pendant qu'un autre vient au tube par des lignes convergentes qu'on nommera matière affluente.»

\section{Pour expliquer les attractions et répulsions d'un corps, Nollet fait appel aux deux courants effluent et affluent:}

«S'il se rencontre donc un corps léger et libre dans le cercle d'activité dont tout l'espace est rempli par la matière tant affluente qu'effluente; l'un et l'autre mouvement étant progressif, ce corps flottant obéit nécessairement au plus fort; il va au tube ou il s'en écarte.»

\section{C'est ainsi, explique-t-il:}

«qu'une petite feuille de métal placée entre deux aigrettes de matière effluente paroit aussi tost attirée précipitament vers le tube, parce qu'en effet la matière affluente l'y conduit».

Quant à la répulsion Nollet l'explique par une augmentation virtuelle de la feuille qui s'est électrisée par communication et de ce fait devient elle-même une source de matière effluente. La feuille ainsi soumise aux rayons effluents du corps électrisé qui ont une grande prise sur elle, est repoussée ${ }^{10}$.

Sa conception sur la constitution intime de la matière électrique est bien exposée dans l'explication qu'il donne des traces lumineuses qui apparaissent sur la pointe des corps fortement électrisés, et de son identification avec le «feu élémentaire».

«Le feu ou la lumière qu'on aperçoit dans les phénomènes de l'électricité, est occasionné par le conflit de la matière effluente du corps électrisé, et de la matière affluente qui vient des autres corps voisins, en sens contraire.» ${ }^{11}$

Et en bon empiriste Nollet poursuit:

«Le choc de deux matières qui se meuvent en sens contraire peut-il être mieux marqué que par la douleur réciproque d'un homme électrisé qui présente son doit à celuy de la personne qui ne l'est point?»

Concernant les deux espèces d'électricité de Du Fay, Nollet les a rejetées en considérant tout simplement l'électricité du verre plus forte que celle des gommes. Il écrit à Jallabert:

«Pour peu que vous méditiez vous concevrez aisément qu'il n’y a pas deux sortes d'électricité.» 
C'est une attitude constante de Nollet. On la retrouvera dans le mémoire qu'il a lu contre les deux espèces d'électricité:

«J'ai été un des premiers à renoncer à la distinction des électricités résineuse et vitrée, et je ne trouve pas mauvais qu'on me désigne comme ayant donné à d'autres l'exemple de cette désertion.» ${ }^{12}$

Dans sa polémique contre les franklinistes qui a commencé à la suite de la connaissance des travaux de Franklin, Nollet écrit à Jallabert le 2 juillet 1751:

«Il y a ici un certain Mr. Dalibard dont les liaisons me sont suspectes.» ${ }^{13}$

(Il fait allusion à l'amitié qui lie Buffon et Dalibard). En qualifiant Dalibard et De Lor de «pauvres physiciens», Nollet rappelle à Jallabert qu'il a déjà trouvé l'identité entre le tonnerre et l'électricité:

«Ouvrez le tome IV de mes leçons de physique, p.314, et vous verrez que je l'ay dit plus de quatre ans avant l'homme à qui l'on s'efforce d'attribuer cette idée.» ${ }^{14}$

Et il ajoute plus loin:

«Il en est de même du pouvoir des pointes qui vous appartient bien légitimement.» ${ }^{15}$

Passant à l'examen des deux électricités en plus et en moins de Franklin, Nollet rappelle qu'il abandonna cette distinction depuis longtemps:

«Il y a plus de quinze ans, aimant mieux attribuer ces effets variables au plus et au moins de force de la matière électrique, occasionnés par la nature des corps frottés et par quelque disposition particulière de l'air ambiant, que d'adopter de nouveaux êtres sans nécessité et sans preuves suffisantes.» ${ }^{16}$

Il confirme sa position vis-à-vis de cette théorie dans sa lettre du 20 décembre 1755:

«J'ai lu à notre rentrée un mémoire contre les électricités en plus et en moins, je n'en aurois pas pris la peine sans que Mr. Le Roy a pris a cœur de faire entrer cette mauvaise physique dans nos mémoires.» ${ }^{17}$

Nollet restera sur ses positions, convaincu de sa théorie de l'effluence et l'affluence simultanées. L'opposition farouche de ses adversaires et l'insistance de plusieurs des «meilleures têtes de l'Académie» l'ont déterminé à réimprimer ses Lettres sur l'électricité, avec quelques adjonctions. Dans sa dernière lettre à Jallabert du 30 juillet 1760, il s'en prend à ses adversaires qui $^{18}$, dit-il: 
«s'efforcent d'établir des notions obscures, des êtres métaphysiques, sur les ruines d'un principe qui me paroit incontestable».

Je voudrais dire quelques mots de l'électrothérapie. A la suite de la découverte de la bouteille de Leyde ${ }^{19}$, qu'il a nommée «l'expérience de Leyde», Nollet a tenté quelques applications sur des paralytiques. C'est avec beaucoup de prudence qu'il a procédé, avec le concours du chirurgien Morand ${ }^{20}$. Nous pouvons comprendre l'hésitation de Nollet, surtout après qu'il ait reçu une lettre de Hollande lui apprenant l'accident qui est arrivé à Doppelmaier ${ }^{21}$. Dans sa lettre à Jallabert, datée du 5 décembre 1746, il lui écrit:

«Je n'électrise plus personne de cette manière qu'avec beaucoup de circonspection. M. Lecat [Professeur de chirurgie à Rouen] qui électrise depuis quelques mois un jeune homme paralytique nous écrit que son malade va de mieux en mieux.»

Les expériences sur les paralytiques se font un peu partout en Europe ${ }^{22}$. Quant à Jallabert, il a déjà procédé à quelques expériences dès 1746, comme le montre sa correspondance avec de Sauvages (François Boissier de Sauvages de la Croix), professeur à Montpellier:

«Grâce à vos instructions, l'électricité est devenue à la mode en cette ville; tout le monde se fait électriser, mais nous ne sommes guère avancés car nous n'avons commencé que depuis peu à travailler, faute de globes.» ${ }^{23}$

Mais déjà, l'électrothérapie ne fait pas l'unanimité de tous les médecins de l'époque. C'est donc avec un certain scepticisme qu'on procède à certaines expériences. Nollet contestera l'affirmation de Jallabert selon laquelle l'électrisation accélère le mouvement du pouls (passant de 80 à 90-96́ par minute). Il écrit dans son mémoire:

«Il se peut que l'électricité ait produit cet effet sur des gens du peuple choisis à l'aventure pour cette opération, qui auraient été effrayés par l'appareil et les phénomènes de l'électricité, mais qu'on expose des physiciens à cette expérience, nous sommes persuadés que l'électrisation n'altérera leur pouls en aucune façon.» ${ }^{24}$

Nollet s'étonnera toujours de ce recrutement de malades pour toutes sortes d'expériences. Il dira la même chose lorsqu'il apprendra la «guérison» des paralytiques de Montpellier. C'est ainsi qu'il écrit, dans sa lettre du 28 avril 1750, en parlant des guérisons de Mr. de Sauvages:

«Plust au Ciel quil ny ait pas d'illusion jen crains toujours quand je vois des faits extraordinaires multipliés sur des gens qui sont pauvres, et qui sçavent qu'on les assistera dès quils auront fixé sur eux l'attention des gens en place.» 
Nollet, qui a obtenu du Ministère de la Guerre l'autorisation d'électriser «cinq ou six paralytiques» aux Invalides, n'a pas eu plus de succès.

«Les paralytiques ne voyant plus de nouveaux progrès qui soutînssent leur patience (car il en faut pour se soumettre à cette espèce de torture) ne se prêtèrent plus qu'avec peine, et en se plaignant. ${ }^{25}$

Une analyse de cette correspondance montre que Nollet avait quelques doutes sur l'efficacité de l'électrothérapie. Sa réserve est encore plus grande après tout ce qu'il a vu durant son voyage en Italie et les observations qu'il a recueillies à propos des intonacatures ${ }^{26}$ :

«Je ne puis m'empêcher de croire que la plupart des guérisons électriques de Turin n'ont été que des ombres passagères qu'on a prises avec un peu trop de précipitation ou de complaisance, pour des réalités.» ${ }^{27}$

Parlant du paralytique de Genève ${ }^{28}$, Nollet écrit:

«La guérison du paralytique de Genève est presque la seule dont je sois bien certain et le peu de succès que j'ai eu aux Invalides [...] me fait craindre que les exemples de paralytiques guéris de cette manière ne soient fort rares à l'avenir.»

Nollet, après avoir mis beaucoup d'espoir dans les applications de l'électricité à la médecine, a été désenchanté et a fini par se résigner:

«Si l'électricité devient jamais un remède en usage, il en sera sans doute de lui, comme de tous les autres dont l'application n'est pas toujours aussi heureuse qu'on le souhaite. Quel est le remède dont les effets sont infaillibles?» ${ }^{29}$

Nollet, dont la théorie de l'effluence et de l'affluence offrait une grande souplesse, a émis des idées qui ont été largement suivies et adoptées par un grand nombre de physiciens de l'époque. Plusieurs savants comme Watson, Musschenbroek et Lesage seront de fidèles disciples jusqu'à la fin de leurs jours. Même ses adversaires ont reconnu ses dons et son talent. Barbeu-Dubourg (1709-1779), que l'on ne peut soupçonner d'être «Nolletiste» a écrit, dans une lettre adressée à M. De Lor, parlant de l'abbé:

«Il seroit à désirer que quelque bon physicien, marchant sur ses traces avec le même zèle et la même habileté, prit à cœur de faire servir les plus brillantes expériences de l'électricité à l'éclaircissement de divers autres points importants de l'œeconomie animale.» ${ }^{30}$

Si nous nous reporterons à l'époque de ses travaux, si nous considérons la faiblesse des effets à constater, les faibles moyens d'observation et de mesure, leur incertitude, les causes diverses qui venaient voiler les lois générales, on éprouve pour ces travaux une véritable admiration. 
${ }^{1}$ Torlais, Jean, L'Abbé Nollet, un physicien au siècle des lumières, Paris 1954. Du même auteur: Une grande controverse scientifique au XVIII' ${ }^{\mathrm{e}}$ siècle, L'abbé Nollet et Benjamin Franklin. In: Revue d'histoire des sciences 9 (Paris 1956) 339-349; Lecot, Victor Lucien Sulpice (abbé), L'Abbé Nollet de Pimprez, Noyon 1856; Grandjean de Fouchy, Eloge de M. l'abbé Nollet. In: Histoire de l'Académie Royale des Sciences, Paris 1770, p. 221-251.

${ }^{2}$ Robinet, René, L'École royale du Génie de Mézières (1748-1794). In: Revue d'histoire des sciences 2 (Paris 1949) 267-270.

${ }^{3}$ Senebier, Jean, Histoire littéraire de Genève, t. 3, Genève 1786, p.126-133; Montet, Albert de, Dictionnaire biographique des Genevois et des Vaudois, 2 vol., Lausanne 1877-1878, vol.2, p.4-6; Nouvelle biographie générale (sous la direction du Dr F. Hoefer, 46 vol., Paris 1857-1866), t. 26, 1861, p.301-302; Galiffe, Jacques-Augustin, Notices généalogiques sur les familles genevoises, 7 vol., Genève 1829-1908 (réimpression, Genève 1976), p.273. (Galiffe donne 1713 à la place de 1712 pour la date de naissance de Jallabert, et Poggendorff, t. 1, p.1188, cite «Louis» à la place de «Jean».) Voir aussi: Wolf, Rudolf, Biographien zur Kulturgeschichte der Schweiz, 4 vol., Zürich 1858-1862, vol.4, p.149-160.

${ }^{4}$ Ms. Jallabert, S. H. 243, fol. 2-66, Bibliothèque publique et universitaire, Genève (18 lettres de Daniel Bernoulli; correspondance 1738-1750).

${ }^{5}$ Nollet, Jean-Antoine, Conjectures sur les causes de l'électricité des corps. In: Mémoires de l'Académie Royale des Sciences, Paris 1745, p. 149-214.

${ }^{6}$ Nollet, Leçons de Physique Expérimentale, 6 vol. in-12, Paris 1743-1764 (7 réimpressions du Tome I et 9 réimpressions du Tome II).

${ }^{7}$ Nollet a toujours cherché à vulgariser la science et à propager les idées de la physique de son siècle. Dans cette préface, il écrit: «Plus occupé du soin de me faire entendre, que du reproche qu'on me pourroit faire d'avoir abandonné le langage des sciences dont il est assez ordinaire de se parer, je tâche de parler et d'écrire comme ont fait avant moi quantité d'auteurs reconnus pour bons, et dont les ouvrages pour la plupart peuvent être mis entre le[s] mains de tout le monde.»

Nollet, loc. cit.t. 1, p.XVII.

${ }^{8}$ Genève a été, au XVIII ${ }^{\mathrm{e}}$ siècle, le centre d'une activité scientifique très intense et a eu une concentration d'éminents savants. Voir Cléopâtre Montandon, Le développement de la science à Genève aux $X V I I I^{\mathrm{e}}$ et $X I X^{\mathrm{e}}$ siècles - le cas d'une communauté scientifique, Vevey 1975. Du même auteur: Sciences et société à Genève aux $\mathrm{XVIII}^{\mathrm{e}}$ et $\mathrm{XIX}^{\mathrm{e}}$ siècles, Gesnerus 32 (1975), p.16-34. On consultera également l'article «Genève» de l'Encyclopédie, ou Dictionnaire raisonné des sciences, des arts et des métiers (par Diderot et d'Alembert), 35 vol., Paris et Neuchâtel 1751-1780, p.577: «Toutes les Sciences et presque tous les Arts ont été si bien cultivés à Genève, qu'on seroit surpris de voir la liste des savans et des artistes en tout genre que cette ville a produits depuis deux siècles.»

9 Nollet a déjà énoncé tous ces principes dans le mémoire que nous avons mentionné (note 5), p. 194-196.

${ }^{10}$ Figure (voir p. 233):

Lettre du 19 Juillet 1745

${ }^{11}$ Par «feu élémentaire» Nollet entend: «le principe et la cause de tous les feux, [...] un fluide subtil présent partout» (Leçons, t.IV, p. 187). 


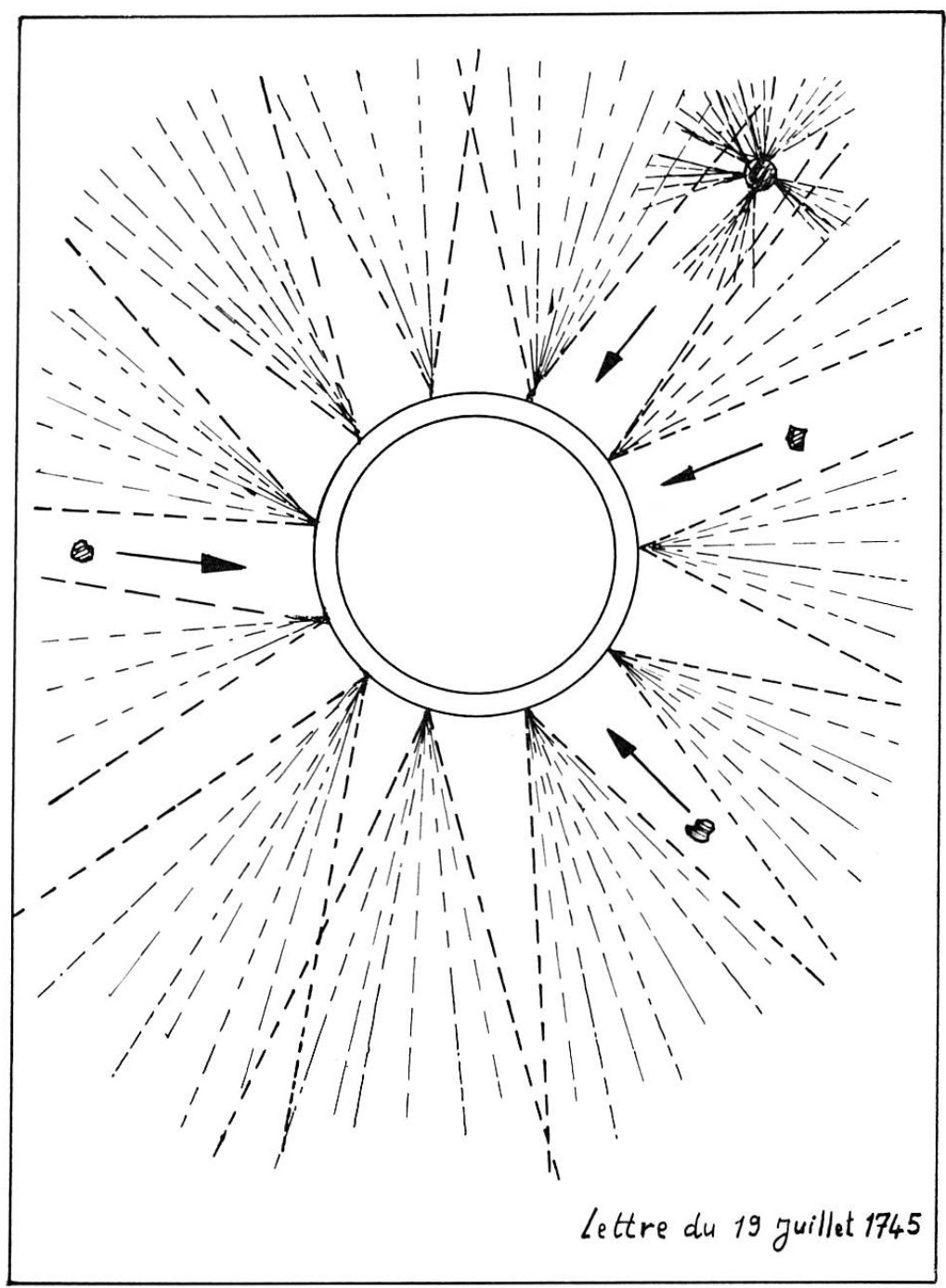

Voir également Roderick W.Home, Nollet and Boerhaave: A note on eighteenth-century ideas about electricity and fire, Annals of Science 36 (1979), no 2, p.171-175; et William M. Sudduth, Eighteenth-century identifications of electricity with phlogiston, Ambix 25 (1978), Part II, p. 131-143.

12 Nollet, Mémoires de l'Académie Royale des Sciences, Paris 1746, p. 12. Voir également Nollet, Essai sur l'électricité des corps, $3^{\mathrm{e}}$ éd., Paris 1753 , p. 436-437.

${ }^{13}$ Dalibard (1703-1779) qui se lia d'amitié avec Buffon qu'il rencontra à Angers, a traduit les Expériences et observations sur l'électricité faites à Philadelphie, en Amérique, par B. Franklin et communiquées dans plusieurs lettres à M.P.Collinson, Paris 1752, 2ème éd.1756. Dalibard a joint à cette traduction une Histoire abrégée de l'électricité, Paris 1752 , où le nom de Nollet ne figure point. Une première explication se trouverait dans la querelle qui opposa Buffon et Réaumur. Buffon n'a jamais pardonné à Réaumur d'avoir été l'inspirateur des Lettres à un Amériquain dont l'auteur est l'Abbé de Lignac (1710-1762). 
Voir à ce propos Maurice Trembley, Correspondance inédite entre Réaumur et Abraham Trembley, Genève 1943, p.362, n. 1; Jean Torlais, L'abbé Nollet et la physique expérimentale au XVIII ${ }^{\mathrm{e}}$ siècle, Paris 1959, Coll. Conférences du Palais de la Découverte, Paris, série D; 60.

14 «Si quelqu'un entreprenoit de prouver par une comparaison bien suivie des phénomènes, que le tonnerre est entre les mains de la nature, ce que l'électricité est entre les nôtres [...]; et que tout dépend du même mécanisme.» (Nollet, Leçons... [voir note 6], t. IV, p. 314.)

15 Jallabert avait déjà fait quelques observations sur les propriétés des pointes dans son livre Expériences sur l'électricité, avec quelques conjectures sur la cause de ses effets, Genève 1748. Le phénomène des pointes observé par Jallabert est décrit dans l'ouvrage de Nollet, Recherches sur les causes particulières des phénomènes électriques et sur les effets nuisibles ou avantageux qu'on peut attendre, Paris 1753, p.312-313; Nollet, Lettres sur l'Electricité..., Paris 1753, Sixième lettre à M. Franklin, p. 129; Pierre Brunet, Les origines du paratonnerre. In: Revue d'histoire des sciences 1 (Paris 1948) 213-253.

${ }^{16}$ Nollet, Suite du Mémoire dans lequel j'ai entrepris d'examiner si l'on est bien fondé à distinguer des électricités en plus et en moins, résineuse et vitrée comme autant d'espèces différentes. In: Mémoires de l'Académie Royale des Sciences, Paris 1755, p.443; Yamazaki, Eizo, L'Abbé Nollet et Benjamin Franklin [...]. In: Japanese studies in the history of science, 1976, no 15, p. 37-62.

${ }^{17}$ Le Roy, Jean-Baptiste, Mémoire sur l'électricité résineuse où l'on montre qu'elle est réellement distincte de l'électricité vitrée, comme feu M. Du Fay l'avait avancé; et qu'elle nous fournit de nouvelles lumières sur les causes de l'électricité naturelle et du tonnerre. In: Mémoires de l'Académie Royale des Sciences, Paris 1755, p.393. Dans ce Mémoire, Le Roy appellera «électricité par augmentation de densité» l'électricité vitrée de Du Fay.

${ }^{18}$ Parmi eux figure Niccola Bammacaro, professeur de philosophie à Naples, dont Nollet réfuta le Tentamen de vi electrica eiusque phaenomenis, Naples 1748.

19 Torlais, Jean, Qui a inventé la bouteille de Leyde? In: Revue d'histoire des sciences 16 (Paris 1963) 211-219; Heilbron, John L., A propos de la bouteille de Leyde. In: Revue d'histoire des sciences 19 (Paris 1966) 133-142. Voir également Heilbron, John L., Electricity in the 17th and 18th centuries, University of California Press 1979, p. 309-323.

${ }^{20}$ Il s'agit d'un paralytique des deux mains. Les résultats de ces expériences ne seront pas concluants et des expériences nouvelles seront tentées une seconde fois en 1748. Nollet a cru obtenir quelques résultats en augmentant l'électrisation pour rendre la commotion plus importante dans «l'expérience de Leyde». (Nollet, Mémoires de l'Académie Royale des Sciences, Paris 1746, p. 28; Nollet, Recherches... [voir note ${ }^{15}$ ], p.404.)

${ }^{21}$ L'accident qui fit mourir Doppelmaier était attribué aux expériences électriques que celuici avait faites sur sa propre personne. Nollet a reçu une lettre de M.Bose de Wittenberg, datée du 15 mars 1747, qui lui apprend «que ce bruit n'avait aucun fondement». La mort de Doppelmaier serait due à une attaque d'apoplexie. Voir Nollet, loc. cit. p.415.

${ }^{22}$ Ladame, P[aul-Louis], Notice historique sur l'Électrothérapie à son origine. L'Électricité médicale à Genève au XVIII ${ }^{\mathrm{e}}$ siècle. In: Revue médicale de la Suisse romande 5, No 10, 15 octobre 1885, p.553-572; No11, 15 novembre 1885, p.625-656; No 12, 15 décembre 1885, p. 697-717.

${ }^{23}$ Ms. Jallabert, 82; fol.44 r., Bibliothèque publique et universitaire, Genève (17 lettres de de Sauvages à Jallabert, correspondance 1743-1750).

${ }^{24}$ Nollet termine son mémoire en disant: «La même expérience répétée sur M. Morand 
[chirurgien] et par d'autres ne nous a rien appris de plus à cet égard.»

Morand et Nollet, Expériences de l'électricité appliquée à des paralytiques. In: Mémoires de l' Académie Royale des Sciences, Paris 1749, p. 56 (Mémoire lu par Nollet).

${ }^{25}$ Nollet, Recherches... [voir note ${ }^{15}$ ], Paris 1753, p.411-412.

${ }^{26}$ Il s'agit de globes ou cylindres en verre contenant diverses drogues appropriées à l'état du malade, fermés hermétiquement. Certains médecins italiens prétendaient avoir guéri les malades par le frottement de ces vaisseaux.

${ }^{27}$ Nollet, Expériences et observations faites en différens endroits de l'Italie. In: Mémoires de l'Académie Royale des Sciences, Paris 1749, p.643-705; cf. Nollet, Essai... [voir note ${ }^{12}$ ], $3^{\mathrm{e}}$ éd., Paris 1753, p.221. (Nollet donne toute une liste des publications qui ont été faites en Italie au sujet des «Intonacatures».)

${ }^{28}$ Il s'agit d'un nommé Noguès, âgé de 52 ans, paralytiques du bras droit. (Ladame indique qu'il s'agit de Noguier âgé de 54 ans, loc. cit. p. 50.)

Jallabert, Jean, Journal de quelques expériences faites sur un paralytique. In: Expériences sur l'électricité, Genève 1748, p.127-135.

${ }^{29}$ Nollet, Recherches... [voir note ${ }^{15}$ ], p.414.

${ }^{30}$ Barbeu-Dubourg, Lettre du traducteur à M. De Lor [professeur de physique expérimentale à Paris]. Parallèle des théories de Franklin et de Nollet. In: Euvres de Franklin, Paris 1773, p. 337-338.

\section{Summary}

Having briefly sketched the life and the work of the two scientists Jean-Antoine Nollet (1700-1770) and Jean Jallabert (1712-1768), the author examines this through unpublished correspondence which covers a period of more than twenty years, certain aspects of Nollet's theory on electricity and its development, as well as certain applications of electricity in medicine, especially in the field of electrotherapy in Geneva.

Isaac Benguigui

Département de Physique théorique

32, Bd. d'Yvoy

CH-1211 Genève 